\title{
Endoscopic Third Ventriculostomy with or without Choroid Plexus Coagulation for Treatment of Hydrocephalus in Guinea: Analysis of 76 Cases in the Department of Neurosurgery of Kipe, Conakry
}

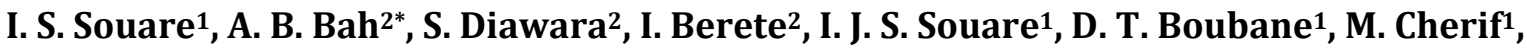 \\ A. M. Camara ${ }^{2}$, L. K. Beavogui ${ }^{2}$
}

${ }^{1}$ Service de Neurochirurgie, Hôpital de l'Amitié Sino-Guinéenne de Kipe, Conakry, Guinea

${ }^{2}$ Service de Neurochirurgie, Hôpital National Donka, Conakry, Guinea

Email: *cushing.bah@gmail.com

How to cite this paper: Souare, I.S., Bah, A.B., Diawara, S., Berete, I., Souare, I.J.S., Boubane, D.T., Cherif, M., Camara, A.M. and Beavogui, L.K. (2021) Endoscopic Third Ventriculostomy with or without Choroid Plexus Coagulation for Treatment of Hydrocephalus in Guinea: Analysis of 76 Cases in the Department of Neurosurgery of Kipe, Conakry. Open Journal of Modern Neurosurgery, 11, 242-251.

https://doi.org/10.4236/ojmn.2021.114028

Received: August 13, 2021

Accepted: October 9, 2021

Published: October 12, 2021

Copyright () 2021 by author(s) and Scientific Research Publishing Inc. This work is licensed under the Creative Commons Attribution International License (CC BY 4.0).

http://creativecommons.org/licenses/by/4.0/ (c) (i) Open Access

\begin{abstract}
In low-income countries, endoscopic third ventriculostomy (ETV) with or without choroid plexus coagulation (CPC) is an increasingly accepted alternative to shunt therapy in adult and pediatric hydrocephalus. The authors report the result of this treatment in Conakry in a mixed population of adult and pediatric patients regardless of the etiology of the hydrocephalus. A retrospective study was conducted on 76 patients undergoing 89 ETV from January 2013 to September 2020. The predominant group of patients was infants less than one year with a mean age of 4.3 months and extremes of $1-8$ months. The H/F sex ratio was $1.7 / 1$. All patients presented acutely with signs of high intracranial pressure. Post-infectious causes and malformations and tumors were the main etiologies, respectively $21 \%, 47.3 \%$, and $15.7 \%$. The mean duration of the endoscopic procedures was $49.93 \pm 10.9 \mathrm{~mm}$, associated with a choroid plexus coagulation in $42 \%$ of cases. The complication rate in the first month was $22 \%$, with CSF leak (5\%) and death (11\%) accounting for the majority. At three months, the complications rates were $45 \%$, with $14.4 \%$ closed stroma, $6 \%$ epilepsy, and $24 \%$ mortality. The mean follow-up was 28 months (range 2 - 53), and the global success rate of $61 \%$. Our study, with its limitations, shows that ETV with CPC is a safe primary approach for the treatment of hydrocephalus in low-income countries regardless of the etiology and the age of the patients.
\end{abstract}




\section{Keywords}

Hydrocephalus, Endoscopic Third Ventriculostomy, Choroid Plexus

Coagulation, Low-Income Countries

\section{Introduction}

Hydrocephalus is the most frequent cause of referral in Pediatric Neurosurgery in Guinea, and most cases of hydrocephalus in children are congenital or acquired during the early childhood period [1]. The ventriculoperitoneal shunt is the traditional hydrocephalus treatment and has shown good outcomes in terms of survival and neurocognitive development [2]. Endoscopic third ventriculostomy (ETV) has recently emerged as a good alternative for obstructive hydrocephalus in most neurosurgical services, with reasonable success rates published in the literature worldwide in adults [3] and infants older than one year regardless of the etiology of the obstructive hydrocephalus [4]. The studies from Warf et al. in the 2000' on the expansion of ETV indications to younger infants with communicating hydrocephalus showed promising results for low-income countries where avoiding shunt dependence is desirable [4] [5]. In Guinea, we adopted ETV with or without choroid plexus coagulation (CPC) since 2012 as a $1^{\text {st }}$ attempt when technically feasible in all cases of hydrocephalus regardless of age and etiology. This approach aimed to reduce CSF shunt dependency and improve clinical outcomes. We, therefore, felt it appropriate, after nine years, to highlight the experience of our department in the practice of this endoscopic technique in the present study.

\section{Patients and Methods}

This was a descriptive retrospective study with seven years eight months from January 2013 to September 2020, conducted at the Hospital of Kipe in Conakry, Guinea. From 2012 to 2014, the national health department set up a hydrocephalus program in this specific hospital with a dedicated team of trained neurosurgeons, nurses, pediatricians, eight beds and a state-of-the-art Karl Storz neuroendoscope system were offered. An NGO financed all the cost of the care, and patients and families were treated for free during this period. Guinea is a low-income country, and neurosurgery is still a new surgical specialty in the environment. Moreover, there is no universal healthcare insurance and not a single Intensive care unit in the entire country.

All patients operated by Endoscopic Third Ventriculostomy (ETV) with or without Choroid Plexus Coagulation (CPC) were included for this study. Patients with incomplete records and previous shunt therapy were excluded. The following data were recorded: age, sex, clinical notes, etiology, previous ETV, intraoperative complications.

The technique of endoscopic perforation of the floor of the ventricle tech- 
nique was standard, using a flexible endoscope pushed further down to perforate the Lilliquist membrane in the prepontine cistern, and the CPC was based on the description by Warf et al., a thermal technique using monopolar coagulation [6].

Outcomes were recorded as complications occurring at one month, three months, and the global success rate was assessed at the final follow-up examination in-person and by phone interview according to the location of the family and its means to travel to the city of Conakry. Eleven families were joined on the phone to evaluate their clinical and functional status and whether the patient was alive. We extracted the remainder data from the record of the last follow-up examination of the patient in the clinical registry.

The success of the ETV was defined as a clinical improvement of the symptomatology after surgery in the absence of any complications requiring a shunt placement at the follow-up evaluation. Failure was defined as the recurrence or worsening of the clinical symptoms of hydrocephalus, a shunt or an external drain placement, any adverse chronic neurologic dysfunction related to the procedure, and death of the patient.

Qualitative variables were expressed in terms of numbers and percentages, and quantitative data in terms of averages with their respective standard deviation sometimes presented in tabular form. Data were collected using the Kobocollect application, and analysis was done on the SPSS software version 25.

\section{Results}

During the study period, 98 patients benefited from 111 ETV, representing $26.88 \%$ of all cases of hydrocephalus operated in the department. Three patients were excluded due to previous shunt placement 19 patients had incomplete records. Seventy-six patients who had undergone $89 \mathrm{ETV}$ were retained for the study, and $56.67 \%$ of the procedures were realized in the first 18 months of the study period (Figure 1).

The predominant group of patients was infants less than one year with a mean age of 4.3 months and extremes of 1 - 8 months; and infants less than two years with a mean age of 17.82 months and extremes of $12-20$ months (Table 1). The $\mathrm{H} / \mathrm{F}$ sex ratio was $1.7 / 1$.

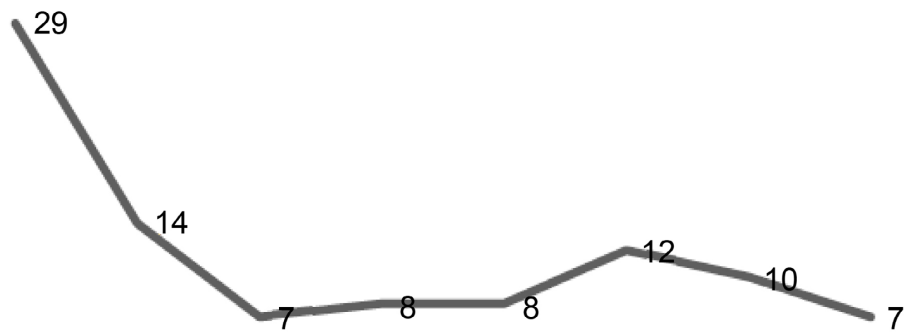

$\begin{array}{llllllll}2013 & 2014 & 2015 & 2016 & 2017 & 2018 & 2019 & 2020\end{array}$

Figure 1. Annual cases of ETV from 2013 to 2020. 
Table 1. Demographic characteristics: more than half of the patients were aged less than one year.

\begin{tabular}{ccc}
\hline Age range & Mean & No of patients $(\%)$ \\
\hline$<10$ months & 4.3 (range $1-8)$ months & $56.5 \%$ \\
$10-24$ months & 17.8 (range $12-20)$ months & $14.4 \%$ \\
$2-18$ years & 7.47 (range 3 - 15) years & $19.7 \%$ \\
$>18$ years & 35 (range 19 - 55) years & $9.2 \%$ \\
\hline
\end{tabular}

All patients presented acutely with signs of extremely high intracranial pressure. Post-infectious causes, malformations, and tumors were the main etiologies, respectively $21 \%, 47.3 \%$, and $15.7 \%$ (Table 2 ).

The mean duration of the endoscopic procedures was $49.93 \pm 10.9 \mathrm{~mm}$. A CPC was associated with the ETV in $42 \%$ of cases. During the operations, we noted primary aqueductal stenosis in 18 patients (23.6\%), a closed Monroe foramen in 8 cases $(10.5 \%)$, thick $3^{\text {rd }}$ ventricle floor in 24 cases $(31.5 \%)$ with hemosiderin deposit in 15 cases (19.7\%), 10 cases of tumor (13.1\%) and hypotrophic choroid plexus in 56 cases (73.6\%). In addition, we found a fenestrated or absent septum pellucidum in 36 cases (47.2\%) and bloody CSF in 14 cases (18.4\%). One thallamostriate vein injury with more moderate bleeding was noted and taken care of by irrigation with isotonic saline fluids.

In the first postoperative month, complications were observed in $22 \%$ of patients with CSF leak (5\%) and death (11\%), accounting for the majority (Table 3). At three months, the complications rates were $45 \%$, with $14.4 \%$ of closed stroma or membrane development below the floor requiring a repeat ETV, 6\% of epilepsy, and $24 \%$ of mortality.

The final follow-up examination time was variable, with a mean of 28 months (range 2 - 53) and a global success rate of $61 \%$. The success rate of repeat ETV was $46.15 \%(\mathrm{~N}=6)$. Among the 6 cases of repeat ETV success, 2 cases were from malformative etiology (33.3\%), and 4 cases were from post-infectious hydrocephalus (66.6\%) (Table 4).

\section{Discussion}

Shunt dependency and associated morbidity is a significant problem in low-income countries like Guinea, as the patients do not have easy access to emergent neurosurgical evaluation and treatment in case of dysfunction or infection [1]. Therefore, finding a way to treat these infants without the use of a shunt is desirable. In this quest, ETV traditionally appears to be a good alternative in obstructive hydrocephalus and infant aged $>2$ years [7]. However, the long-term outcomes in infants lower than one year and other etiologies of hydrocephalus are still in dispute [8].

The first attempt of endoscopic CPC to address this issue in children was first published in 1970 by Scarff et al. in 39 children with 67\% success [9]. In 1995, another large series of 104 patients treated with CPC showed a significantly 
Table 2. Causes of hydrocephalus in the series: malformations were the most prominent etiology.

\begin{tabular}{lc}
\hline \multicolumn{1}{c}{ Etiology } & No of patients (\%) \\
\hline Malformation & $36(47.3 \%)$ \\
- Dandy-Walker (DW) & $18(23.6 \%)$ \\
- Myelomeningocele (MM) & $12(15.6 \%)$ \\
- Primary aqueductal stenosis (PAS) & $06(7.8 \%)$ \\
Tumor & $11(15.7 \%)$ \\
- Posterior fossa tumor & $6(7.8 \%)$ \\
- Craniopharyngioma & $1(13 \%)$ \\
- Pineal tumor & $2(2.6 \%)$ \\
- Third ventricle tumor & $3(3.9 \%)$ \\
Post-infectious & $16(21 \%)$ \\
Post-hemorrhage & $1(1.3 \%)$ \\
Unknown & $11(14.4 \%)$ \\
Total & 76 \\
\hline
\end{tabular}

Table 3. Postoperative complications.

\begin{tabular}{ccc}
\hline Complications & 1 month & 3 months \\
\hline CSF leak & $4(5 \%)$ & 0 \\
Subarachnoid hemorrhage & $2(3 \%)$ & 0 \\
Closed stoma & $2(3 \%)$ & $11(15 \%)$ \\
Epilepsy & 0 & $5(6 \%)$ \\
Mortality & $8(11 \%)$ & $18(24 \%)$ \\
\hline
\end{tabular}

Table 4. ETV success rates according to age group and causes of hydrocephalus: ETV had a good success rate in all age and etiologic group except hemorrhage (there was only one case in the whole cohort) and infections.

\begin{tabular}{|c|c|c|}
\hline \multicolumn{2}{|c|}{ Factor } & ETV Success \\
\hline \multirow{4}{*}{ Age } & $<10$ months & $70.6 \%$ \\
\hline & $10-24$ months & $77.8 \%$ \\
\hline & $2-18$ years & $66.7 \%$ \\
\hline & $>18$ years & $66.7 \%$ \\
\hline \multirow{4}{*}{ Etiology } & Malformation (36) & $72 \%$ \\
\hline & Tumor (11) & $60 \%$ \\
\hline & Infection (16) & $26 \%$ \\
\hline & Hemorrhage (1) & $0 \%$ \\
\hline
\end{tabular}

higher success rate in infants with communicating hydrocephalus [10]. However, due to methodological flaws and technological limitations of these early studies, this treatment modality declined in favor of the shunts.

In the 2000', Benjamin, C, Warf, and colleagues in Uganda did a series of investigations in East Africa. They postulated in their preliminary results that a CPC has an added benefit when associated with ETV in infants younger than 
one year of age with myelomeningocele, post-infectious hydrocephalus, Dandy-Walker malformation, and communicating hydrocephalus [11]. They defended its use by the complementary effect between the pulsation reducing property of CPC and the pulsation absorber property of the ETV [4]. As a result, ETV/CPC was proposed as an effective primary treatment option [11]. Since these landmark observations, some African neurosurgery teams followed this trend, offering ETV with or without CPC in hydrocephalus as a primary treatment option regardless of age and etiology, provided that the CSF is not purulent or acutely hemorrhagic [12] [13].

The number of cases included in our series is modest compared to neighboring countries with comparable patient demographics and the same study period [13] [14] [15]. Our study is notable for the uneven distribution of the annual number of cases. More than half of the patients included in the series were operated in the first 2 years of the study period. During this period, the program was well funded, the patients were actively recruited from all neonatal care department in the country with massive communications to counteract traditional cultural assumptions about extreme macrocrania in infants [1]. These patients were investigated and treated for free and followed up appropriately in the postoperative period. In 2014, the hospital was deserted since it was the epicenter of the Ebola pandemic [16]. In 2015 the program was stopped, and the subvention for the complete care of patients was drastically reduced. Since then, the number of cases has significantly dropped in the department. This phenomenon reflects the challenge in developing neurosurgery care and research in an environment where patients are not financially covered by an insurance system [17].

The mean age observed in our study relates to the diverse nature of our study population (adult and pediatric). In the first two years of the study period, the recruitment was almost exclusively pediatric, and $69.9 \%$ of the patients were less than two years old. The male predominance is generally found in studies where congenital hydrocephalus is more prevalent due to $\mathrm{X}$ recessive mode of transmission [18]. The prevalence of DW malformation, myelomeningocele, aqueduct stenosis, and post-infectious communicating hydrocephalus in the pediatric subgroup of our series is comparable to most sub-Saharan African studies [11] [14] [15]. This is different from high-income countries where posthemorrhagic hydrocephalus, in premature patients that have survived a neonatal intraventricular hemorrhage episode, is more prevalent. This difference in etiologic profile between low income and high-income countries has been one of the many reasons why the findings of Dr. Warf's team have not been primarily adopted by north American pediatric neurosurgeons in their practice [19] [20].

ETV/CPC is a short procedure. The incidence of low-abundance bleeding from subependymal vessels ranges from $1 \%$ to $3 \%$ in the literature. In all these cases, bleeding which does not interfere with the performance of the ETV is controlled by irrigation with normal saline or cauterization with the coagulating probe [21]. In this series, 2 cases of bleeding of low abundance are reported 
(2.63\%). In 1 case, it was bleeding from the edges of the stoma, and in the other case, it was bleeding from an injured intraventricular vessel that required the coagulating probe's use. In both cases, transient irrigation was sufficient to control bleeding.

Ten patients (14\%) experienced ETV-related complications during the follow-up, and 4 of them had a CSF leak (5\%). Most of them occurred in our first year of experience, reflecting the learning curve of the procedure. We later adopted a smaller dural opening and a more watertight closure of the wound. It is a well-described complication of ETV with an incidence in the literature ranging from $0.7 \%$ to $7 \%$ of cases [22]. They are usually addressed by repetitive subtractive lumbar punctures revealing the possibility of a progressive adaptation of the hydrodynamics of the LCS through the stoma [21]. The subtractive puncture was performed in 4 patients associated with suturing of the leak. The other case of complications was late-onset epilepsy, and we couldn't tell if it was a real consequence of the ETV, the hydrocephalus disease itself, or related to other conditions like poor feeding or dehydration. No evidence of infectious or endocrine complications was observed. ETV is known to have a lower incidence of infectious complications than shunt [23].

The mortality in our series was $35 \%$ at the final follow-up, and all death cases occurred after the discharge. Whether or not the death is directly linked to the surgical procedure or external factors like other medical conditions and the difficult access to a hospital from remote areas of the country remains unknown. A previous report in the same hospital found a mortality of $41 \%$ for shunt therapy [1].

In infants of $1-24$ months, the ETV success rate was superior to that of other age groups. While the hypothesis of ETV having an acceptably good outcome has been defended, it is not known to be more beneficial in infants than adults [20]. It should be considered that mean follow up in this group was shorter than the general study population, 14 months (range 4 -19). Older children and adults were more accessible to follow up than young kids coming from very remote areas.

The overall success rate of ETV in our series is comparable to the literature [13] [21] [24]. In the series from Bankole et al. in Nigeria, the combination of ETV/CPC has avoided a shunt placement in $75 \%$ of the study group [13]. In our cases, out of 76 patients, at a follow-up of 28 months, 46 patients were shunt-free and alive. 7 patients have been shunted for the failure of a repeat ETV and 26 patients have died.

The main questions to ask from the perspective of resource-limited areas are:

1) Is ETV/CPC a dangerous procedure? The answer is no in well-trained hands with appropriate materials, according to our experience and many authors [4].

2) Is the procedure harmful? Evidence in the present study and most studies in Africa shows low morbidity [12] [14]. However, some questions have been 
raised regarding the effect of coagulating the $\mathrm{CPC}$ on the protein content of the CSF and the future neurocognitive development of the children [19] [20]. In a complex environment like ours, the definition of meaningful clinical success is different than that of a higher-income country. With more elaborated studies from "wealthier" west African countries like Senegal, Cote d'Ivoire, or Nigeria, longer follow-up can be ambitioned with serial neurocognitive assessment.

3) Does its use as a primary treatment option reduce the effectiveness of a subsequent shunt? There is no evidence that ETV/CPC increases the risk of subsequent shunt failure for patients who ultimately need shunt placement after stoma closure. It avoids shunt-related problems in patients who don't fail, which is a significant achievement in our area.

Considering these facts and our results, we retain ETV and CPC when feasible as a primary treatment modality in all pediatric hydrocephalus and obstructive hydrocephalus cases in adults.

Our study has many limitations, from the general methodological flaws of a retrospective study to the specific financial and logistic challenges of doing neurosurgery clinical research in limited resources environments. For example, we couldn't correctly analyze the mortality rate in our series, which is multifactorial, and we could have easily got a much longer follow-up had the non-governmental funding program continued, to define better the neurocognitive well-being of kids younger than one year treated by ETV and CPC regardless of the etiology. As for the success rate, much ETV failure occurs in the first six months, which is covered by our follow-up period [21].

\section{Conclusion}

The result from this study in Guinea suggests that primary ETV with or without CPC and a second attempt in case of failure and when it's feasible, is an acceptable approach in terms of success rate and safety, weighted with the benefit of sparing a significant portion of our patients the complications and struggles of the long term follow up of intracranial shunts. According to the literature, a subsequent shunt placement following failure of an ETV appears to have comparable outcome with a primary shunt placement [21]. The decrease in our hospital affluence following the interruption of the NGO financing of the whole care underlines further the necessity of implementing a national insurance coverage, at least for the pediatric population.

\section{Conflicts of Interest}

The authors declare no conflicts of interest regarding the publication of this paper.

\section{References}

[1] Souare, I.S., Beavogui, L.K., Bah, A.B., Camara, S.N., et al. (2016) Hydrocephalus in the Pediatric Population in the Tropic Co-Morbidity Impact at CHU in Conakry. American Journal of Psychiatry and Neuroscience, 4, 65-70. 
https://doi.org/10.11648/j.ajpn.20160404.12

[2] Souare, I.S., Fofana, A., Atakla, H.G. and Souare, I.S.J. (2021) Complications of Hydrocephalus Surgery in Children: Study of 18 Cases at the University Hospital Center of Conakry. International Journal of Neurosurgery, 5, 62-65.

[3] Choi, J.U., Kim, D.S. and Kim, S.H. (1999) Endoscopic Surgery for Obstructive Hydrocephalus. Yonsei Medical Journal, 40, 600-607. https://doi.org/10.3349/ymj.1999.40.6.600

[4] Warf, B.C. (2005) Comparison of Endoscopic Third Ventriculostomy Alone and Combined with Choroid Plexus Cauterization in Infants Younger than 1 Year of Age: A Prospective Study in 550 African Children. Journal of Neurosurgery, 103, 475-481. https://doi.org/10.3171/ped.2005.103.6.0475

[5] Warf, B.C. (2013) Congenital Idiopathic Hydrocephalus of Infancy: The Results of Treatment by Endoscopic Third Ventriculostomy with or without Choroid Plexus Cauterization and Suggestions for How It Works. Childs Nervous System, 29, 935-940. https://doi.org/10.1007/s00381-013-2072-1

[6] Warf, B.C., Dewan, M. and Mugamba, J. (2011) Management of Dandy-Walker Complex-Associated Infant Hydrocephalus by Combined Endoscopic Third Ventriculostomy and Choroid Plexus Cauterization. Journal of Neurosurgery: Pediatrics, 8, 377-383. https://doi.org/10.3171/2011.7.PEDS1198

[7] Warf, B.C. (2005) Hydrocehlaus in Uganda: The Predominance of Infectious Origin and Primary Management with Endoscopic Third Ventriculostomy. Journal of Neurosurgery, 102, 1-15. https://doi.org/10.3171/ped.2005.102.1.0001

[8] Koch, D. and Wagner, W. (2004) Endoscopic Third Ventriculostomy in Infants of Less than 1 Year of Age: Which Factors Influence the Outcome? Child s Nervous System, 20, 405-411. https://doi.org/10.1007/s00381-004-0958-7

[9] Scarff, J.E. (1970) The Treatment of Nonobstructive (Communicating) Hydrocephalus by Endoscopic Cauterization of the Choroid Plexuses. Journal of Neurosurgery, 33, 1-18. https://doi.org/10.3171/jns.1970.33.1.0001

[10] Pople, I.K. and Ettles, D. (1995) The Role of Endoscopic Choroid Plexus Coagulation in the Management of Hydrocephalus. Neurosurgery, 36, 698-701. https://doi.org/10.1227/00006123-199504000-00009

[11] Warf, B.C. (2013) The Impact of Combined Endoscopic Third Ventriculostomy and Choroid Plexus Cauterization on the Management of Pediatric Hydrocephalus in Developing Countries. World Neurosurgery, 79, S23.e13-5.

https://doi.org/10.1016/j.wneu.2011.02.012

[12] Ndounbe, A., Simeu, C. and Motah, M. (2015) Non-Tumor Obstructive Hydrocephalus Treated with Endoscopic Third Ventriculostomy in Cameroon. Open Journal of Modern Neurosurgery, 5, 137-143. https://doi.org/10.4236/ojmn.2015.54022

[13] Bankole, O.B., Ojo, O.A., Nnadi, M.N., et al. (2015) Early Outcome of Combined Endoscopic Third Ventriculostomy and Choroid Plexus Cauterization in Childhood Hydrocephalus. Journal of Neurosurgery. Pediatrics, 15, 524-528. https://doi.org/10.3171/2014.10.PEDS14228

[14] Mbaye, M., Gahito, L., Thiam, A.B., et al. (2020) The Outcome of Endoscopic Third Ventriculostomy in a Mixed Population of Adult and Pediatric Patients. Open Journal of Modern Neurosurgery, 10, 325-333. https://doi.org/10.4236/ojmn.2020.103035

[15] Salem-Memou, S., Thiam, A.-B., Kpelao, E., et al. (2014) Traitement chirurgical de l'hydrocephalie de l'enfant par ventriculocisternotomie endoscopique au Senegal. Neurochirurgie, 60, 254-257. https://doi.org/10.1016/j.neuchi.2014.06.014 
[16] Chughtai, M., Loua, T.O., Beavogui, K. and Qureishi, A.L. (2015) Neurological Surgery in Guinea, West Africa. Journal of Vascular and Interventional Neurology, 8 , S12-S16.

[17] Rudolson, N., Dewan, M., Park, K.B., et al. (2019) The Economic Consequences of Neurosurgical Disease in Low- and Middle-Income Countries. Journal of Neurosurgery, 130, 1149-1156. https://doi.org/10.3171/2017.12.JNS17281

[18] Kousi, M. and Katsanis, N. (2016) The Genetic Basis of Hydrocephalus. Annual Review of Neuroscience, 39, 15-16.

https://doi.org/10.1146/annurev-neuro-070815-014023

[19] Drake, J. (2012) Endoscopic Third Ventriculostomy. Journal of Neurosurgery Pediatrics, 10, 461-462. https://doi.org/10.3171/2012.6.PEDS12230

[20] Souweidane, M.S. (2014) Combined Choroid Plexus Coagulation and Endoscopic Third Ventriculostomy: Is North America Ready? Journal of Neurosurgery Pediatrics, 14, 221-223. https://doi.org/10.3171/2014.4.PEDS1450

[21] Sacko, O., Bortto, S., Lauwers, C., Dupuy, M. and Roux, F. (2010) Endoscopic Third Ventriculostomy: Outcome Analysis in 368 Procedures. Journal of Neurosurgery. Pediatrics, 58, 8-15. https://doi.org/10.3171/2009.8.PEDS08108

[22] Erşahin, Y. and Arslan, D. (2008) Complications of Endoscopic Third Ventriculostomy. Child s Nervous System, 24, 16-18.

[23] Whitehead, W.E. and Kestle, J.R. (2001) The Treatment of Cerebrospinal Fluid Shunt Infections. Results from a Practice Survey of the American Society of Pediatric Neurosurgeons. Pediatric Neurosurgery, 35, 5-10.

https://doi.org/10.1159/000050422

[24] Warf, B.C., Mugamba, J. and Kulkarni, A.V. (2010) Endoscopic Third Ventriculostomy in the Treatment of Childhood Hydrocephalus in Uganda: Report of a Scoring System That Predicts Success. Journal of Neurosurgery. Pediatrics, 5, 143-148. https://doi.org/10.3171/2009.9.PEDS09196 